\title{
3 Research Square \\ Symptomatic Discal Pseudocyst after Percutaneous Endoscopic Lumbar Discectomy: 3 Case reports and retrospective review analysis
}

Hai-ting Wu ( $\nabla$ wht1551@163.com)

Ningbo No 2 Hospital: Ningbo Huamei Hospital University of Chinese Academy of Sciences Yun Wang

Ningbo No 2 Hospital: Ningbo Huamei Hospital University of Chinese Academy of Sciences

\section{Research Article}

Keywords: percutaneous endoscopy, discectomy, pseudocyst, surgical complications

Posted Date: May 4th, 2021

DOl: https://doi.org/10.21203/rs.3.rs-456135/v1

License: (c) (1) This work is licensed under a Creative Commons Attribution 4.0 International License.

Read Full License 


\section{Abstract}

Objective To investigate the clinical features and treatment of post-discectomy symptomatic Discal Pseudocyst after percutaneous endoscopic lumbar discectomy.

Methods A retrospective analysis of the data of 3 patients with concurrent symptomatic discal pseudocyst after percutaneous endoscopic lumbar discectomy revealed that all patients were female and had unilateral radiculopathy due to single-segmental lumbar disc herniation. Symptoms were alleviated obviously after the first operation, but pains recurred and even worse than before. The mean recurrence time was 5 weeks (2-8 weeks). Post-operative MRI showed quasi-circular occupancy in the original surgical area (low signal on T1-weighted image, high signal on T2-weighted image, same as cerebrospinal fluid), and communicating with the intervertebral disc.

Results The symptoms of two patients gradually alleviated after conservative treatment, and the followup MRI showed that the cyst had completely absorbed within one year. Another patient underwent percutaneous endoscopic transforaminal cyst excision under local anesthesia again. Round-like tumors were found in the operation, with dark red and viscous contents. Postoperative cyst wall pathology suggested fibrous cyst wall tissue, and postoperative symptoms were relieved immediately. Cystic space occupancy disappeared after 6 months follow-up.

Conclusion The concurrent symptomatic discal pseudocyst after percutaneous endoscopic lumbar discectomy are rare and often occur in the early postoperative period, and can be clearly diagnosed by MRI examination. Patients who fail to respond to conservative treatment may require surgical treatment and have a good prognosis.

\section{Introduction}

Pseudocyst of intervertebral disc is a cystic lesion in the surgical area of intervertebral disc, which often leads to recurrence or even aggravation of preoperative symptoms after compressing nerve root. Its morphology and imaging manifestations are similar to those of disc cysts, which are cystic masses formed on the surface of the disc and often contain bloody fluid [1, 2]. Generally, Magnetic Resonance Imaging(MRI) in the early postoperative period of the patients showed hyperintense T2-weighted image and hypointense T1-weighted image in the surgical area of the primarily fibrous ring, the surface of the protruding fibrous ring, and even process to the foramen or spinal canal. Unlike general cysts, it has a shorter formation time and often occur within 1 month after surgery, their structures communicate with the intervertebral disc. Enhanced MRI can show incomplete ring-shaped enhancement areas, and incomplete cyst wall structure can also be found after surgical resection, so it is called pseudocyst [3].

The incidence of symptomatic discal pseudocyst is extremely low and tends to occur after microscopic, discoscopic, and foraminoscopic minimally invasive surgery, whereas Kang and Park [3] report an incidence of approximately $1 \%$, and most patients are young men, with preoperative MRI showing mild degeneration of the surgical segment of the disc. However, there is still insufficient understanding of its 
pathogenesis and natural course, and there is also controversy about whether to carry out surgical treatment. To this end, we report 3 cases of concurrent symptomatic discal pseudocyst after percutaneous spinal endoscopy, and review the relevant literature to explore the clinical features, diagnostic points and treatment of pseudocyst of intervertebral disc.

\section{Clinical Data}

From November 2016 to September 2019, 412 patients with percutaneous endoscopic lumbar discecetomy (PELD) were enrolled in our hospital, including 197 males and 215 females, with an average age of $52.38+16.7$ years. Surgery indications included lumbar disc herniation (LDH)and lumbar spinal stenosis. The range is from L3-4 to L5-S1, all of which are single-segment.

Surgical approach: All PELD surgeries were performed by 2 experienced spine surgeons. Local anesthesia via the intervertebral foramen approach was used in all segments of $L 4 / 5$ and above, transforaminal approach was used in 58 cases of L5/S1, and general anesthesia through the vertebral plate approach was used in 117 cases due to high iliac spine and other reasons.

Take the $L 4 / 5$ lateral transforaminal approach through the intervertebral foramen under local anesthesia as an example: make the patient prone, and pad pillows on the chest and iliac part of the patient to keep the abdomen suspended. The needle insertion point was marked 6-13 cm apart according to the preoperative imaging data and the patient's body size. After local anesthesia was satisfactory, C-armguided puncture was performed to target the ventral part of the superior articular process apex and personalized adjustment was made according to the situation of nucleus pulposus protrusion. Cut the skin about $8 \mathrm{~mm}$ at the needle insertion point, and place the dilatation tube step by step along the guide wire. If necessary, enlarge the intervertebral foramen with a circular saw, establish a working channel, place the endoscope after the fluoroscopy is satisfactory, and continue to lavage with the lavage solution, explore the nerve root under the microscope, and relieve the compression. The nerve root pulsed well without obvious compression, and the patient felt that the symptoms had improved significantly. Therefore, hemostasis, withdrawal from the working system, and selective placement of drainage tube according to the bleeding situation in the surgical field, suture the incision.

\section{Result}

The symptoms of 412 patients were significantly improved and discharged within 1 week. Three patients had recurrent symptoms or even worsening pain in the follow-up, all of which occurred in the short term. (Tab.1).The follow-up data were as follows:

Case 1. The patient was a 60-year-old female who was admitted to the hospital for "Low back pain and left leg radicular pain for more than 2 years,aggravation for 3 days".

Physical examination: straight-leg-raising tests of left leg restricted to $20^{\circ} \otimes$ Manual muscle testing of the left extensor hallucis longus and the left tibialis anterior was grade 4. Radiological examination: Lumbar 
CT and MRI showed L4/5 disc herniation with secondary spinal canal stenosis (Fig. 1). Diagnosis: Lumbar disc herniation (L4/5), lumbar spinal canal stenosis(LSS). On December 6, 2019, PELD via transforaminal approach were performed under local anesthesia, and the symptoms were significantly improved. From 2 weeks after surgery, the patient developed radiation pain in the posterolateral thigh of the left lower limb, which was more severe than that before surgery and affected sleep. Outpatient reexamination of MRI showed high signal on T2-weighted images at the original operative segment, low signal on T1-weighted images with a circular shape of $10 \times 6 \mathrm{~mm}$, compressing the left L5 nerve root, and no relief was observed after bed rest and oral administration of non-steroidal anti-inflammatory and analgesic drugs. Therefore, on January 12, 2020, the "Lumbar Endoscopic Exploratory Cleaning + Cyst excision" operation was performed again under local anesthesia. During the process, a red encapsulated cystic mass was found on the ventral side of the left L5 nerve root, which was completely excised, and the cystic fluid was dark red bloody gelatinous liquid. Postoperative pain symptoms improved, and the pathological results of cyst wall examination suggested fibrous cyst wall tissue.(Fig. 2).

Case 2: Female, 27yrs. This patient was admitted to the hospital due to "low back pain with right lower limb pain and numbness for more than 3 months". Physical examination: Lasegue sign and Bragard sign of right lower limb was positive.Manual muscle testing of the right extensor hallucis longus and the left tibialis anterior was grade 4. visual analogue scale (VAS):4 points. Diagnosis: Lumbar disc herniation (L4/5). (Fig. 3).

The patient received PELD surgery successfully under local anesthesia on May 23, 2019. No severe complications were occurred during operation. The VAS score for radiating pain in the right lower limb at discharge was 1 point. However, symptoms were recurred 1 month post-operation, mainly radiating pain in the right lower limb, and VAS score was 3 points. The patient was given conservative treatment such as swelling and pain relief, but the symptoms did not be relieved. CT and MRI were reexamined 42 days after the operation. The results showed that there was a cystic space below the level of the L4/5 intervertebral space. Continue the above-mentioned conservative treatment. L-MRI was reexamined 54 days after the operation. The results showed that the cystic space was slightly enlarged, the VAS score was 3 points, and the muscle strength in the right lower extremity did not decrease. After 3 months after the operation, the patient's symptoms gradually alleviated. Lumbar CT results showed that the volume of the cystic space was reduced. 1 year after the operation, the patient was followed up on $\mathrm{MRI}$, and the results showed that the cystic space was absorbed. (Fig. 4).

Case 3. Female,15Yrs,suffered from"Low back pain with left leg radicular pain for 6 months,aggravation for 3 days".

Physical examination: Lasegue sign and Bragard sign of left lower limb was positive.Manual muscle testing of the extensor hallucis longus and the left tibialis anterior was grade 3. VAS:5 points. Diagnosis: Lumbar disc herniation (L5/S1).

This patient received PELD surgery successfully under local anesthesia on June 8,2020 . The patient improved and was discharged 3 days after operation. The VAS score for radiating pain in the left lower 
limb at discharge was 1 point. Symptoms were recurred 2 month post-operation, mainly radiating pain in the left lower limb, and VAS score was 3 points.

\section{Discussion}

Young et al first reported 2 cases of cysts after nucleus pulposus enucleation in 2009, which were distinguished from disc cysts because of incomplete cyst wall and named post-discectomy pseudocyst (PDP) [4]. PDP is a rare complication after discectomy, which usually occurs about 1 month after the initial operation and is prone to occur in young male patients with mild disc degeneration $[2,5]$. Kang et al.[3] reported that the incidence of PDP after PELD was 1\% (15 cases of PDP occurred after PELD in 1503 patients). The cysts are mostly located around the posterolateral longitudinal ligament of the dura mater and are oval in shape. The volume of the cysts depends on the surgical residual lacunae, and the inner boundary of the cyst wall rarely passes through the midline of the intervertebral disc. However, the incidence of PDP reported by Ryutaro et al.[6] was only $0.28 \%$ (2 PDP occurred in 359 patients after PELD surgery). Cystic space occupancy can stimulate peripheral nerve roots and sinus vertebral nerves, leading to low back pain, lower extremity radiation pain or sarcomere paralysis weakness. These clinical symptoms are similar to disc recurrence and residual nucleus pulposus, which are difficult to identify by symptoms and signs.

Chiba et al.[7] first named the intraspinal extramedullary cyst in which the contents of the cyst communicated with the intervertebral disc as discalcyst in 2001. Shiboi et al.[8] later wrote that PDP also has the characteristics of disc cysts: (1) predisposition to Asian populations, especially young men; (2) spinal nerve root symptoms similar to disc herniation; (3) mild degeneration of the involved disc; (4) discography confirmed that the cyst is pedunculated with the disc; (5) cystic fluid is mostly bloody fluid or serum; (6) pathological section found that the cyst wall was mostly fibrous connective tissue, lacking of intervertebral disc tissue and epithelial cells, and inflammatory cell infiltration or inflammatory granulation tissue proliferation, sometimes hemosiderin deposition. Considering so many similarities between PDP and disc cysts, we believe that the two may be homologous diseases, while PDP is a special type of disc cysts, and the pathogenesis of both have many similarities.

The pathogenesis of PDP remains unclear. Toyama [9] and Chiba [7] have proposed a hypothesis that a cyst is formed by disc injury or rupture of intraspinal vessels. Toyama et al. [9] reported that 4 out of 7 cysts were hemorrhagic fluid, which was speculated to be cysts caused by intradisc hemorrhage after disc injury, or epidural hematoma caused by intraspinal venous plexus tear, and hematoma was absorbed into cysts. Chiba et al. [7] retrospectively analyzed 8 cases of intervertebral disc cysts and considered that the formation of cysts was associated with disc injury. Chung and Young et al. [2, 4] put forward another hypothesis, which is that the prominent intervertebral disc induces an inflammatory response, making granulation tissue wrapped around the surface of the annulus fibrosus to form a pseudomembrane, while PELD destroys part of the fibrosus ring in the process of removing the nucleus pulposus, but does not remove the pseudomembrane outside the fibrosus ring, resulting in the axial load of patients after surgery squeezing the fluid and blood extravasation in the disc, thus it forms PDP together with the 
pseudomembranes. Kang et al. [3] believe that electrocoagulation, hemostasis, vaporization and other operations during PELD cause inflammatory reaction, while vertebral plate approach injures the posterior longitudinal ligament, aggravates the inflammatory reaction between the posterior longitudinal ligament, fibrosus ring and surrounding tissues, thus inducing PDP. Kono et al. [10] proposed that the pathological characteristics of cysts are similar to the formation mechanism of meniscal cysts of knee joints and synovial cysts of articular process joints. They believed that the cyst originates from a degenerated intervertebral disc at the surgical segment, and that the fluid spilled from the components of the nucleus pulposus in the disc accumulates in the epidural space through a damaged fibrosus ring, which stimulates inflammatory expression leading to reactive pseudomembranes formation. However, there is still a lack of strong evidence whether pseudocysts are formed by pseudomembranes followed by fluid accumulation or by fluid accumulation followed by pseudomembranes formation.

The treatment of PDP remains controversial. The results of conservative treatment and surgery reported in the literature are satisfactory. Chung et al. [2] reviewed 12 patients with symptomatic discal pseudocyst after surgery, 6 of whom were treated conservatively. The cysts had a long time span with an average spontaneous absorption time of 82.7 day. Therefore, it is believed that postoperative pseudocysts are different from other types of spinal extradural cysts, and because the cyst wall lacking tissue structure is relatively thin and easy to be phagocytosed and absorbed, in the environment of no inflammatory response expression, surgical treatment is recommended for patients with intolerable clinical symptoms, which is the same as Manabe et al. Surgical treatment is recommended as early as possible if there is no obvious relief of conservative treatment in the short term, and even the symptoms worsen and neurological dysfunction occurs, such as muscle weakness, sensory retardation, reflex changes and cauda equina syndrome. Literature review $[12,13]$ CT-guided aspiration therapy has a good short-term follow-up effect, but does not completely destroy the structure of the capsule wall, so there is a high recurrence rate. However, percutaneous endoscopy and microscopy have good surgical field of view, can completely remove cysts and connected prominent discs, low recurrence rate, and can achieve satisfactory clinical efficacy.

The Case 2 and Case 3 were reexamined after conservative treatment, and the cysts disappeared within 1 year, indicating that some PDP cases had self-limitation and self-healing tendency, which may be an important reason for the effective conservative treatment. We believe that there is a self-limiting reason for PDP: PDP is more likely to spontaneously absorb because of the incomplete structure of the cyst wall and thinning as the cyst enlarges. According to the literature summary and our treatment experience: for patients with asymptomatic PDP, periodic review of MRI shows that the cyst can spontaneously subside. For patients with symptomatic PDP, conservative treatment should be taken first, such as oral antiinflammatory and analgesic drugs, dehydration and edema-reducing drugs, and Mecobalamin tablets for the treatment of peripheral nerves. Surgical treatment may be considered for those with no relief or aggravation of symptoms after conservative treatment.

Kang et al. [3] retrospectively analyzed 1503 cases of PELD treatment, including 1118 cases of L4/5 treated by transforaminal approach, 6 cases of PDP occurred, the incidence was $0.54 \%$. In 330 cases of 
L5/S1 treated with PELD via vertebral plate approach, 9 cases of PDP occurred with an incidence of $2.73 \%$, while 55 cases of other segments had no PDP. The results indicated that the vertebral plate approach $(L 5 / S 1)$ may be more prone to PDP $(P<0.05)$. We believe that the occurrence of PDP may not necessarily be related to the surgical lumbar segment, but to the way the operator operates, such as too much separation of the dural sac and ligamentum flavum, too much exposure of the posterior edge of the diseased disc, too much removal of the posterior longitudinal ligament structure, too much tissue interference and damage, may all be responsible for the high incidence of PDP, which is the same as Kang et al.

However, further retrospective analysis and even cohort studies are needed to confirm the causes, pathology and pathogenesis of PDP, which is the direction of our next efforts.

\section{Conclusion}

In conclusion, pseudocyst of intervertebral disc is an early complication after PELD surgery. The etiology, pathology and treatment of pseudocyst are still controversial. It seems valid to attempt conservative treatment first because there is a possibility of spontaneous absorption of cysts.If symptoms continue to worsen and recur, surgical treatment need to be adopted, with a good prognosis.

\section{List Of Abbreviations}

MRI:magnetic resonance imaging

PELD:percutaneous endoscopic lumbar discecetomy

LDH: lumbar disc herniation

CT:computed tomography

LSS:lumbar spinal canal stenosis

PDP:post-discectomy pseudocyst

\section{Declarations}

\section{Ethics approval and consent to participate:}

Not applicable.

\section{Consent for publication}

Yes!

\section{Availability of data and materials}


Yes!

\section{Competing interests}

No.

\section{Funding}

Ningbo natural science foundation (No. 2018A610265).

\section{Authors' contributions}

Not applicable.

\section{Acknowledgements}

All authors have no conflict of interest.

\section{References}

[1] Jha SC, Tonogai I, Higashino K, et al. Postoperative discal cyst: An unusual complication after microendoscopic discectomy in teenagers[J]. Asian J Endosc Surg, 2016, 9(1): 89-92.

[2] Chung D, Cho DC, Sung JK, et al. Retrospective report of symptomatic postoperative discal pseudocyst after lumbar discectomy [J]. Acta Neurochir , 2012, 154(4):715-722.

[3] Kang SH, Park SW. Symptomatic post-discectomy pseudocyst after endoscopic lumbar discectomy[J]. J Korean Neurosurg Soc, 2011, 49(1):31-36.

[4] Young PM,Fenton DS,Czervionke LF. Postoperative annular pseudocyst:report of two cases with an unusual complication after microdiscectomy and successful treatment by percutaneous aspiration and steroid injection[J]. Spine J,2009,9(2): e9-e15.

[5] Subash CJ, Ichiro T, Kosaku H, et al. Postoperative disacl.cyst : an unusual complication after microendoscopic discectomy in teenagers [J] . Asian J Endosc Surg, 2016, 9: 89-92.

[6] Shiboi R, Oshima Y, Kaneko T, et al. Different operative findings of cases predicted to be symptomatic discal pseudocysts after percutaneous endoscopic lumbar discectomy. J Spine Surg. 2017;3(2):233-237.

[7] Chiba K,Toyama Y,Matsumoto M,et al. Intraspinal cyst communicating with the intervertebral disc in the lumbar spine:diseal cyst[J].Spine(Phila Pa 1976),2001,26(19): 2112-2118.

[8] Shiboi R,Oshima Y,Kaneko T,et al. Different operative findings of eases predicted to be symptomatic diseal pseudocysts after percutaneous endoscopic lumbar discectomy[J].J Spine Surg,2017,3(2):233237. 
[9] Toyama Y,Kamata N,Matsumoto M,el al. Pathogenesis and diagnostic title of intraspinal cyst communicating with intervertebral disk in the lumbar spine[J],Rinsho Seikei Geka,1997,32(5):393-400.

[10] Kono $K$, Nakamura $H$, Inoue $Y$, et al. Intraspinal extradural cysts communication with adjacent herniated disk: imaging characteristics and possible pathogenesis [J] . AJNR Am J Neuroradiol, 1999, 20 (7) : 1373-1377.

[11] Manabe H , Higashino K, Sugiura K. A Rare Case of a Discal Cyst Following Percutaneous Endoscopic Lumbar Discectomy via a Transforaminal Approach[J]. International Journal of Spine Surgery, 2019, 13(1):6012.

[12] Erhan A, Irsadi D, Gülcin S, et al. Which treatment method should be preferred for lumbar discal cysts? A case report and a review of the literature [J] . Polish Neurological Scoiety, 2014, 48 (1) :71-75.

[13] Yu HJ, Park CJ, Yim KH. Successful Treatment of a Symptomatic Discal Cyst by Percutaneous C-arm Guided Aspiration. Korean J Pain. 2016;29(2):129-135.

\section{Table}

\section{Table 1.Summary of Patients}

\begin{tabular}{lccccccll}
\hline $\begin{array}{l}\text { Case } \\
\text { No. }\end{array}$ & Age/sex & Level & Side & $\begin{array}{l}\text { Post-op VAS } \\
\text { change }\end{array}$ & Aggravation & MRI(days) & $\begin{array}{l}\text { Contents } \\
\text { of Cyst }\end{array}$ & $\begin{array}{l}\text { Follow- } \\
\text { up(months) }\end{array}$ \\
\hline 1 & $60 / \mathrm{F}$ & L4/5 & L & $5 \rightarrow 1$ & 2 weeks & 30 & $\begin{array}{l}\text { Light } \\
\text { bloody }\end{array}$ & 14 \\
\hline 2 & $27 / F$ & L4/5 & R & $4 \rightarrow 1$ & 1 month & 54 & - & 21 \\
\hline 3 & $15 / F$ & L5/S1 & L & $3 \rightarrow 0$ & 2 month & 113 & - & 8 \\
\hline
\end{tabular}

\section{Figures}



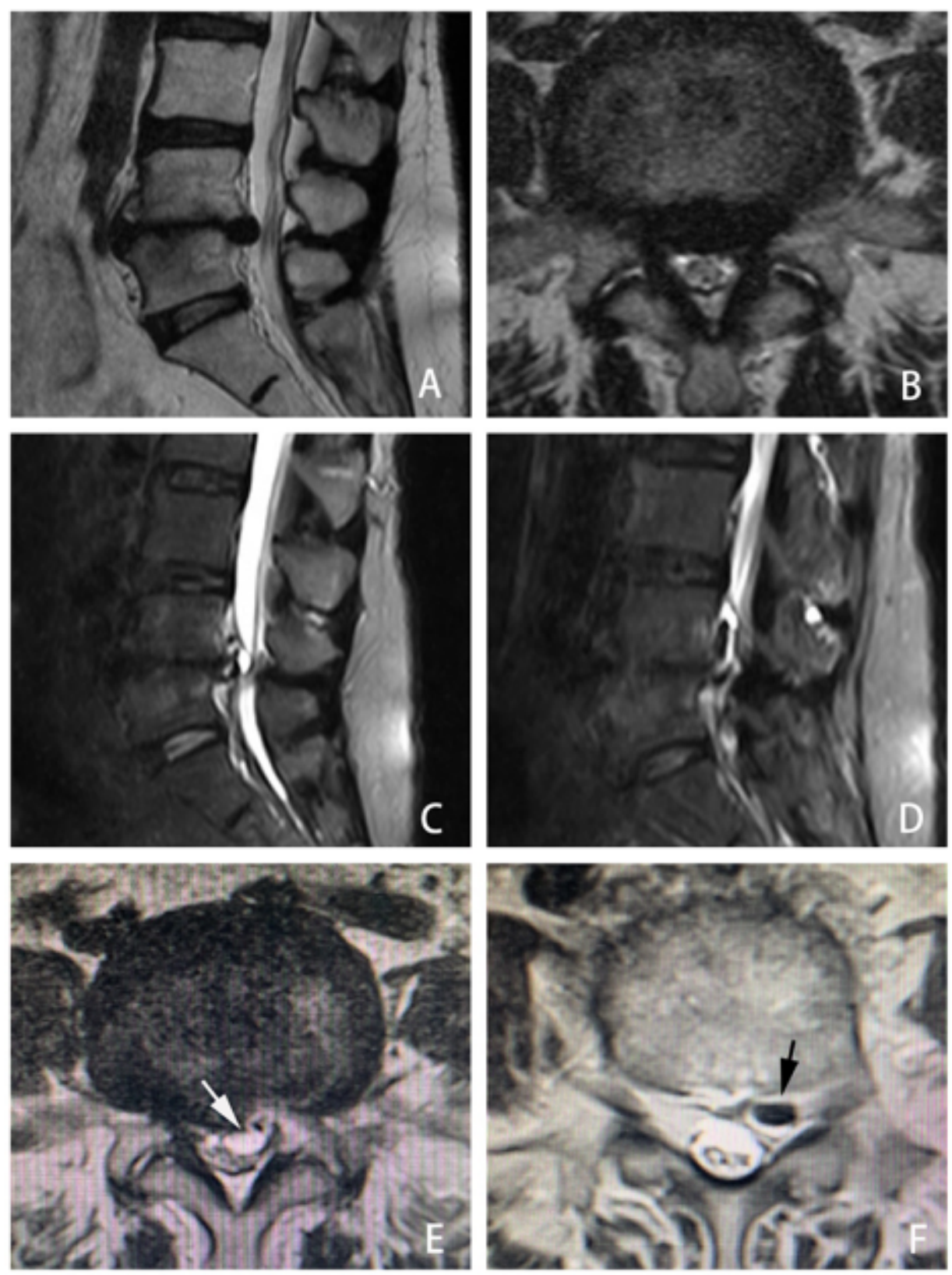

Figure 1

60-year-old female patient, who was admitted to the hospital because of " Low back pain and left leg radicular pain for more than 2 years,aggravation for 3 days ", without symptoms of sciatic nerve in the right lower extremity. Symptomatic discal pseudocysts developed postoperatively. A-B: Preoperative lumbar MRI showed lumbar 4/5 disc herniation, paracentral left-sided herniation, lateral recess stenosis, and compression of dural sac. C-D: Sagittal position of lumbar spine MRI 30 days after surgery, cystic space occupancy appeared in lumbar 4/5 segment. E-F: Transverse position of lumbar spine MRI 30 days after surgery, cystic space occupancy appeared in the lumbar $4 / 5$ segment of the spinal canal and the left intervertebral foramen area. 

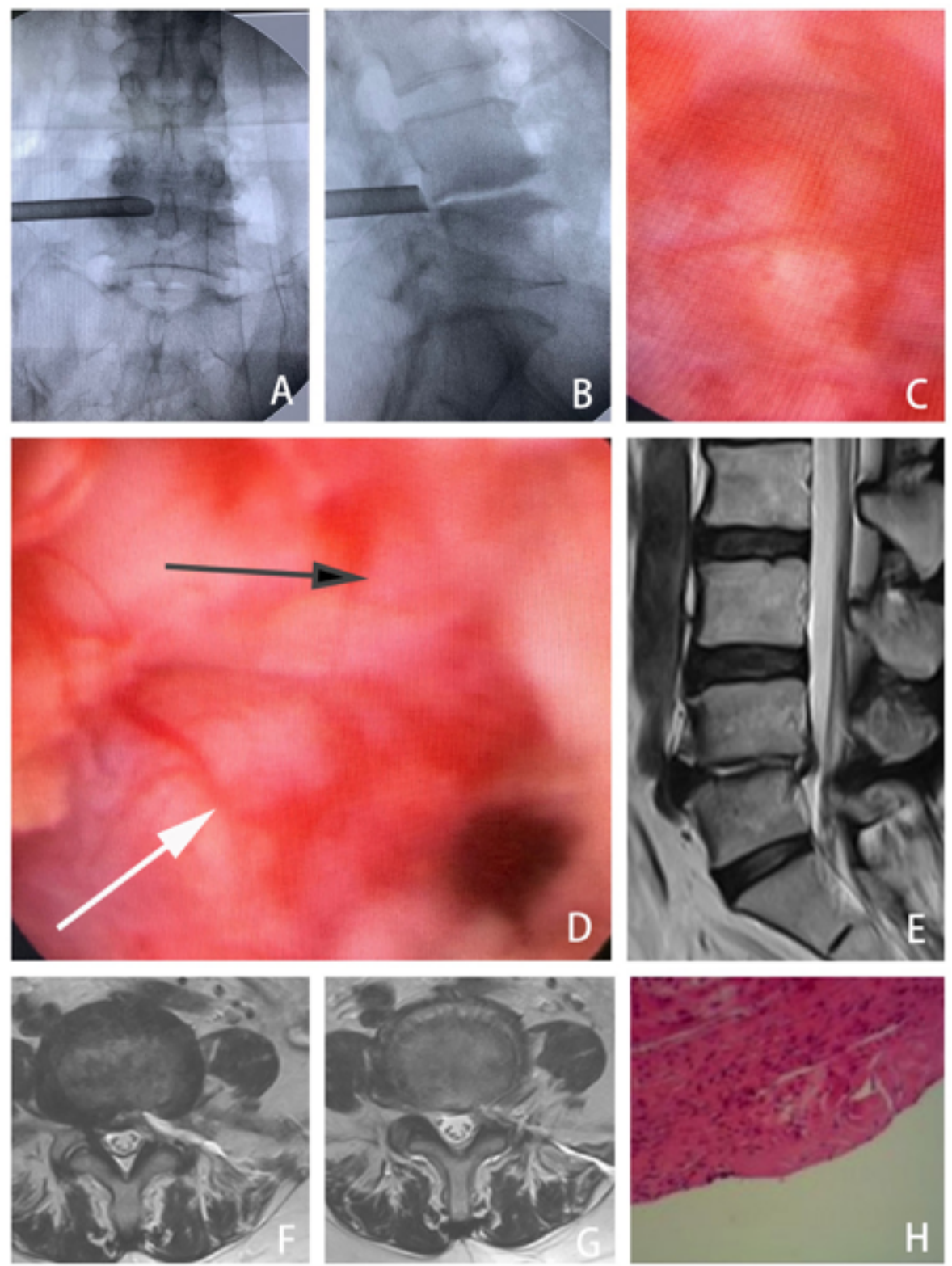

Figure 2

Percutaneous spinal endoscopy revision surgery A-B: C-arm fluoroscopy shows that the working cannula is in good position. C-D: Intraoperative endoscopic exploration of cystic space occupancy, dark red, compression of L5 nerve root. " $\rightarrow$ " denotes L5 nerve root. "White $\rightarrow$ " denotes cystic space occupancy. E-G: Five days after revision surgery,MRI showed that the original cyst had been removed without obvious compression of nerve root and dural sac. $\mathrm{H}$ : Postoperative pathology showed fibrous cyst wall tissue (HE staining, $x$ 100). 

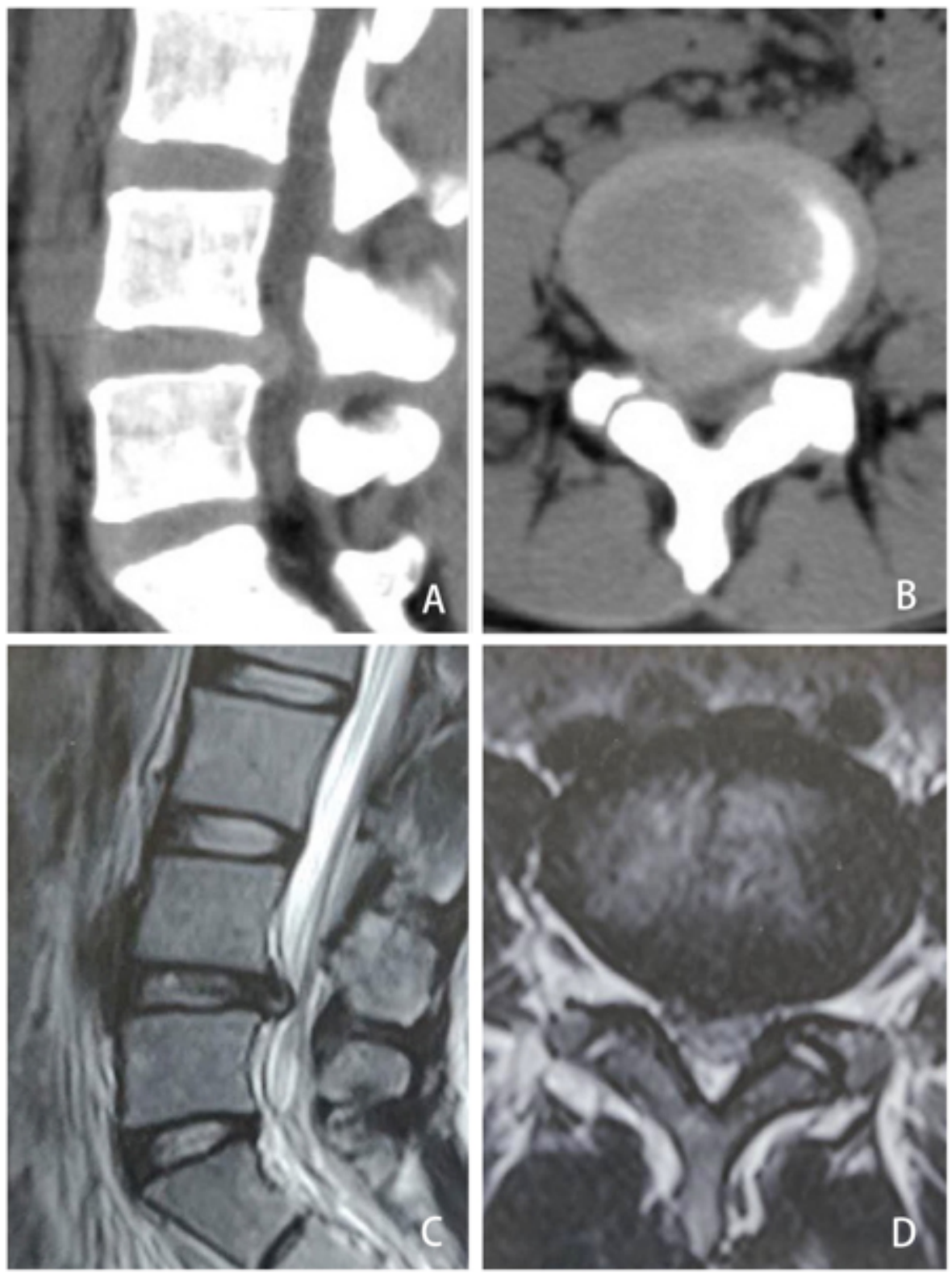

\section{Figure 3}

Female, 27yrs, suffered from "low back pain with right lower limb pain and numbness for more than 3 months". Scoring 4 on the VAS of radiating pain in the right lower limb. Diagnosis: Lumbar disc herniation (L4/5). A-B: Preoperative lumbar CT, C-D: Preoperative lumbar MRI; suggested a lateral central protrusion herniation (right). 

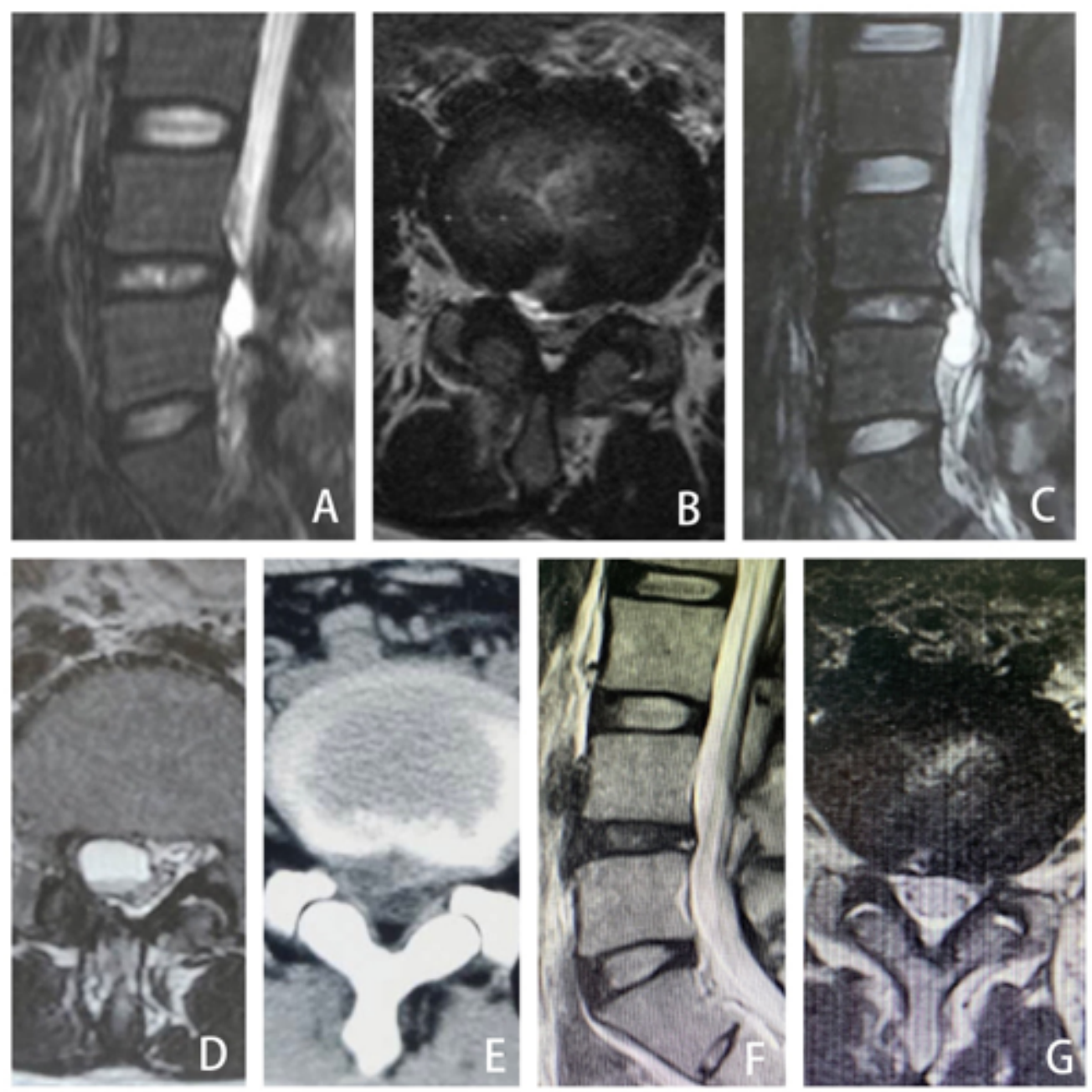

\section{Figure 4}

Follow-up radiological imaging A-B: 42 days after PELD, a cystic space occupancy was seen below the level of L4/5 intervertebral space. C-D: Lumbar MRI showed that the cyst was larger than before(54 days after surgery). E: Lumbar CT reexamined 3 months after operation showed space occupying in lumbar 4/5 spinal canal, and the shape was similar to that of herniated intervertebral disc. F-G: Lumbar MRI showed that cystic space occupancy had been absorbed 14 months after surgery. 

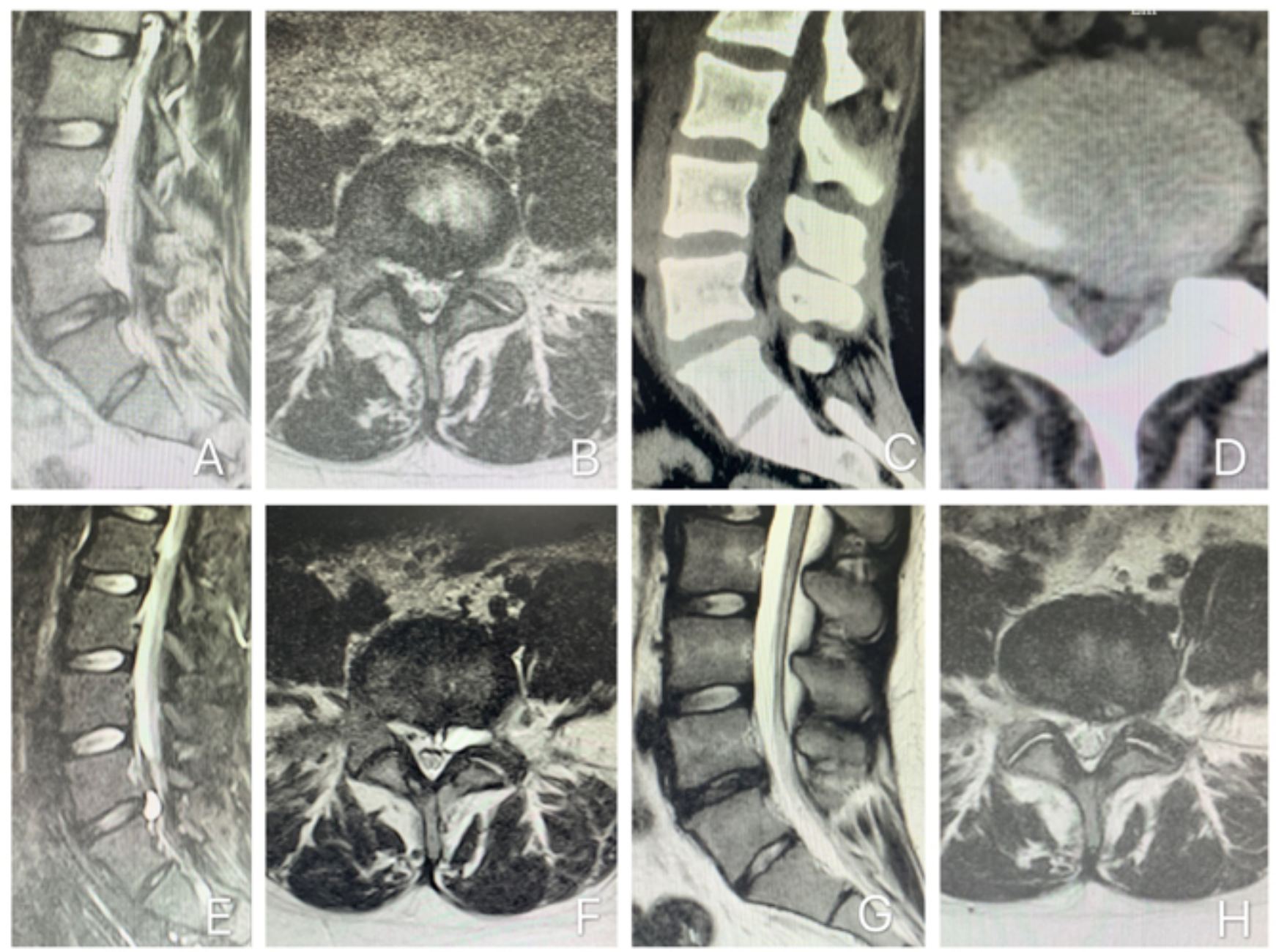

Figure 5

Female,15Yrs,suffered from"Low back pain with left leg radicular pain for 6 months,aggravation for 3 days". Diagnosis: Lumbar disc herniation (L5/S1). A-B: Preoperative lumbar MRI; suggested a lateral central disc herniation. C-D: Lumbar CT scan showed L5/S1 disc herniation,no calcification. E-F: 3 months(Sep.29,2020) after PELD, MRI shows a cystic space occupancy was seen on the level of L5/S1 intervertebral space. G-H: Lumbar MRI showed that cystic space occupancy had been absorbed 6 months after surgery (Jan.1,2021). 